\section{Pseudoquiste pancreático intramural gástrico en páncreas heterotópico, una causa infrecuente de retención gástrica. Caso clínico}

\author{
GIANCARLO SCHIAPPACASSE ${ }^{1,5}$, FABIÁN VILLACRÉS ${ }^{5, a}$, \\ CRISTIÁN CAVALLA ${ }^{2,5}$, FRANCO ORELLANA ${ }^{3,5}$, \\ ALEJANDRA GALLARDO ${ }^{4,5}$, ROBERTO CHARLES ${ }^{2,5}$
}

\section{Gastric intramural pseudocyst in a heterotopic pancreas causing gastric outlet obstruction. Report of one case}

Heterotopic pancreas is a silent gastrointestinal malformation that may become clinically evident when complicated by inflammation and pseudocyst formation. We report a 26 year-old male presenting with vomiting, pain and abdominal distention. An abdominal CT scan showed an important gastric distention secondary to a 4-cm cystic lesion located in the antrum wall. An endosonography showed that the lesion obstructed the gastric outlet and was compatible with a pseudocyst. A cysto-gastrostomy was performed draining the cyst. Its high lipase and amylase content confirmed that it was a pancreatic pseudocyst. Six months later, the lesion appeared again and a subtotal gastrectomy was performed Histopathology confirmed ectopic pancreatic tissue.

(Rev Med Chile 2018; 146: 933-937)

Key words: Gastric Outlet Obstruction; Pancreas; Pancreatic Pseudocyst.
'Departamento de Imágenes. Clínica Alemana de Santiago. Santiago, Chile.

${ }^{2}$ Servicio de Cirugía. Clínica

Alemana. Santiago, Chile.

${ }^{3}$ Servicio de Endoscopia Digestiva.

Clínica Alemana de Santiago.

Santiago, Chile.

${ }^{4}$ Servicio de Anatomía Patológica.

Clínica Alemana de Santiago.

Santiago, Chile.

${ }^{5}$ Facultad de Medicina.

Universidad del Desarrollo-Clínica

Alemana. Santiago, Chile.

aResidente de Radiología.

Los autores declaran no tener conflictos de interés.

Trabajo no recibió finaciamiento.

Recibido el 5 de enero de 2018 , aceptado el 18 de julio de 2018.

Correspondencia a:

Dr. Giancarlo Schiappacasse Departamento de imágenes. Clínica Alemana de Santiago.

Chile.

gschiappacasse@gmail.com
$\mathrm{E}$ 1 páncreas heterotópico o ectópico es una entidad rara, definida como la presencia de tejido pancreático en una ubicación distinta a la habitual, sin continuidad anatómica o vascular con el páncreas y puede ocurrir en cualquier parte del tracto gastrointestinal ${ }^{1}$. En la mayoría de casos el páncreas heterotópico no produce síntomas, pero ocasionalmente puede presentarse como dispepsia, pancreatitis ectópica, hemorragia digestiva alta o como una masa inflamatoria abdominal. Los pseudoquistes pancreáticos pueden ocurrir en localizaciones atípicas como mediastino, riñones o bazo $^{2}$. Son raros los casos reportados en ubicación gástrica con síntomas de obstrucción al vaciamiento gástrico ${ }^{3}$.

Presentamos un caso de síndrome de retención gástrica secundaria a un pseudoquiste pancreático intramural en el antro gástrico secundario a inflamación de tejido pancreático ectópico.

\section{Caso clínico}

Varón de 26 años, sin antecedentes mórbidos personales ni familiares de importancia conocidos, presentó dolor y distensión abdominal asociado a náuseas, vómitos no biliosos y ausencia de expulsión de gases de 5 días de evolución. No presentaba fiebre.

Al examen físico, el abdomen se encontraba distendido, con sensibilidad difusa, sin signos de irritación peritoneal. Sus exámenes de laboratorio al ingreso mostraban un hematocrito de $46 \%$, leucocitos: $10.600 / \mathrm{mm}^{3}$, PCR: $0,5 \mathrm{mg} / \mathrm{dl}$, amila- 

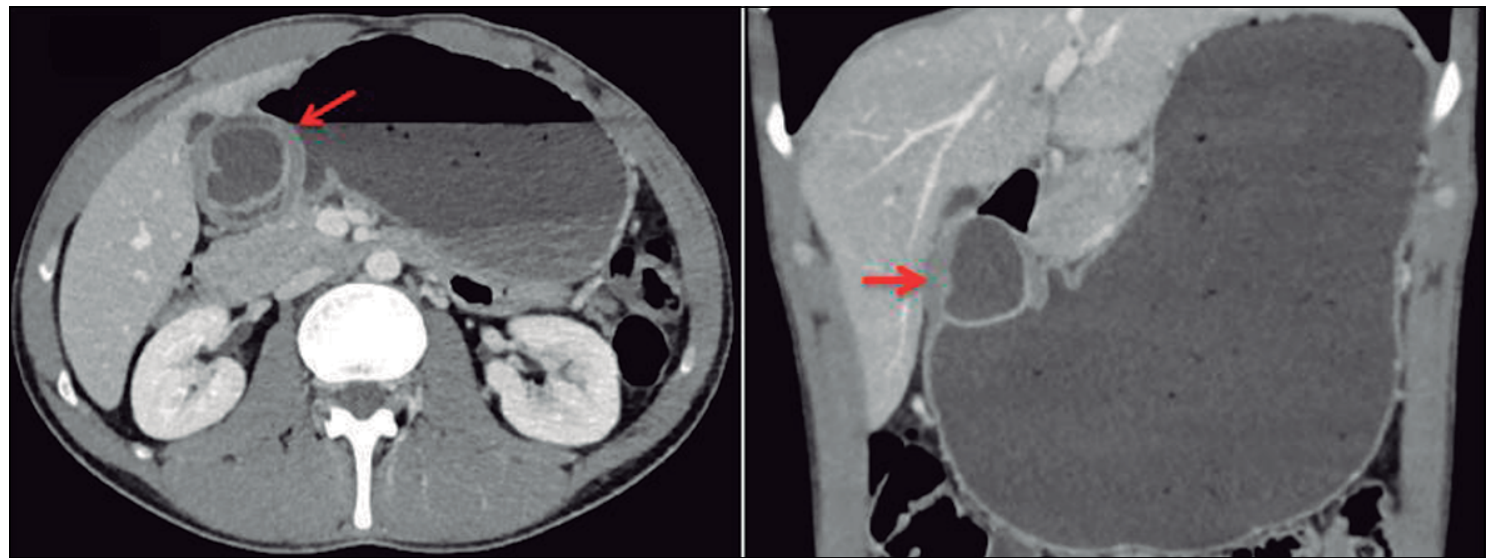

Figura 1. Imágenes de tomografía computada con contraste en cortes axial y coronal que muestran importante distensión gástrica secundaria a lesión quística en región de antro gástrico (flechas rojas).

semia $131 \mathrm{U} / \mathrm{L}(\mathrm{VN}: 28-100)$ y lipasemia $129 \mathrm{U} / \mathrm{L}$ (VN: 13-60).

Se realizó una tomografía de abdomen y pelvis que demostró importante distensión gástrica con contenido heterogéneo secundario a lesión quística en el espesor de la pared del antro gástrico de aproximadamente $4 \mathrm{~cm}$. No se observaban signos de neumatosis parietal gástrica ni perforación (Figura 1). En vista de los hallazgos, se procedió a realizar una endosonografía que evidenció, a nivel de la cara antero-superior de la región antro-pilórica, una lesión solevantada de aproximadamente $4 \mathrm{~cm}$, con mucosa que la recubría de aspecto normal, determinaba estenosis infranqueable del píloro (Figura 2). Al ultrasonido endoscópico se observó una lesión hipoecogénica, bien delimitada, de pared fina, con escaso flujo al Doppler color de 3,5 x 3,1 cm dentro de las eco-capas de la mucosa gástrica, compatible con pseudoquiste (Figura 3). El pseudoquiste fue posteriormente puncionado con aguja 19 (Accesss needle, Cook Medical) bajo visión endosonográfica, dando salida a líquido sero-hemático (Figura 4). Se realizó una cistogastrostomía con cistogastrostomo (Cook Medical) y se destechó la lesión con papilotomo de punta y pulltype, drenando abundante líquido sero-hemático, quedando la cavidad sin contenido (Figura 5). El resultado del estudio bioquímico del líquido del pseudoquiste fue alto contenido en lipasa: $57.930 \mathrm{U} / \mathrm{L}$ y amilasa: 1.971 U/L, lo que confirmó que se trataba de un pseudoquiste pancreático intramural en el antro gástrico, secundario a pancreatitis en tejido pancreático heterotópico. El paciente mejoró sus síntomas y fue controlado a las $24 \mathrm{~h}$ posteriores al drenaje con una tomografía computada con contraste oral que demostró adecuado paso de contraste a intestino delgado y ausencia de filtraciones a cavidad

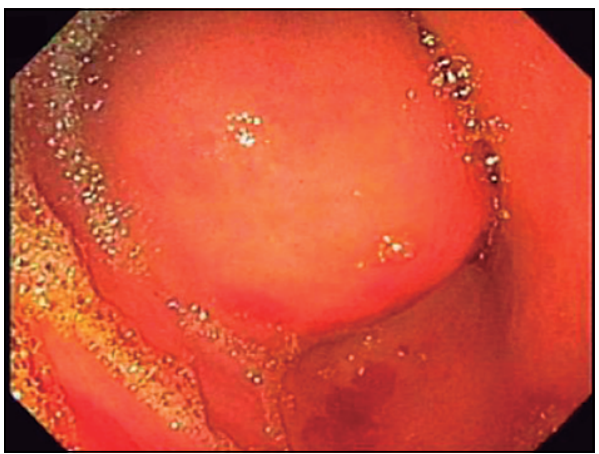

Figura 2. Endoscopia evidencia una lesión solevantada de aproximadamente $4 \mathrm{~cm}$ en región antro-pilórica.

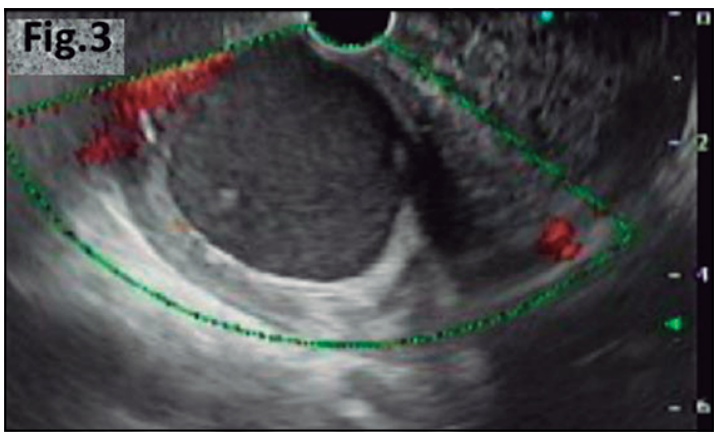

Figura 3. Endosonografía muestra una lesión hipoecogénica bien delimitada dentro de las eco-capas de la pared gástrica. 


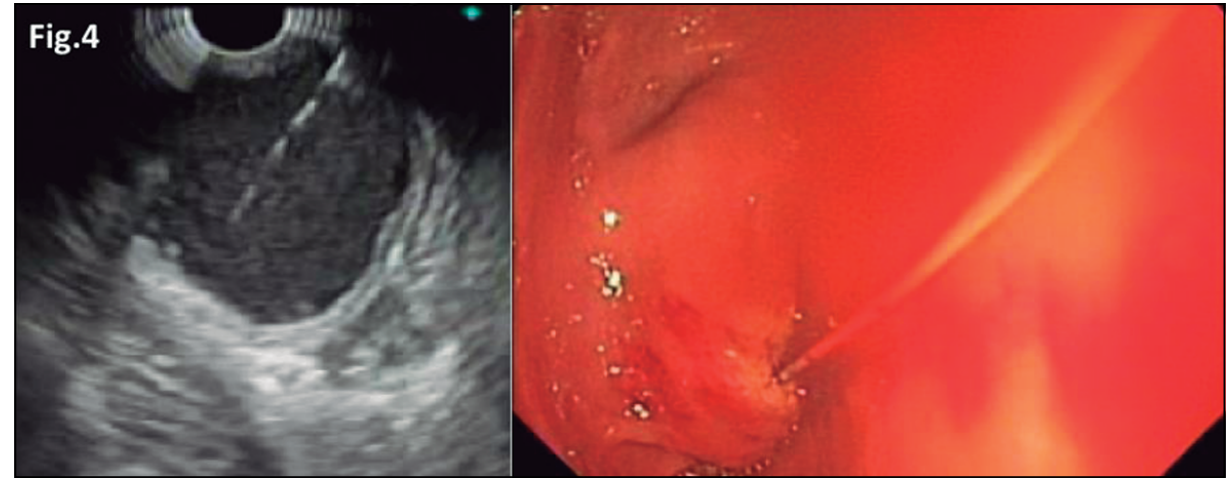

Figura 4. Punción guiada por endosonografía.

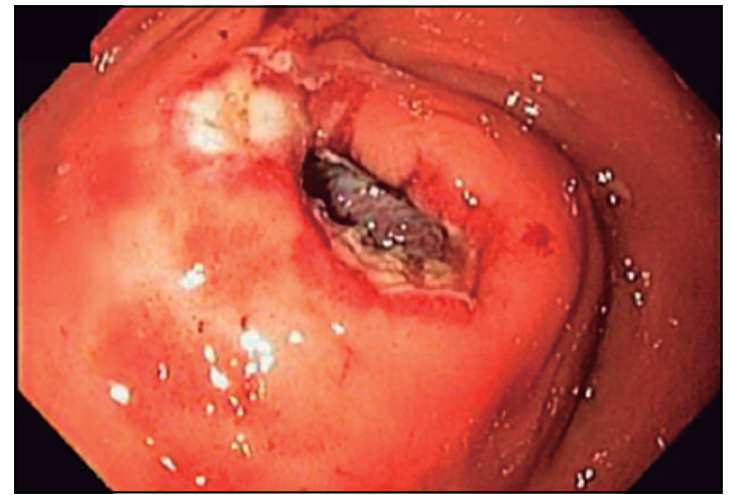

Figura 5. Cistogastrostomía y destechamiento de la lesión, la cual quedó sin contenido.

abdominal y en un control 3 meses después, se encontraba asintomático con una imagen residual de $1 \mathrm{~cm}$ (Figura 6). El paciente presentó síntomas abdominales 6 meses después y en un control tomográfico se observó que el pseudoquiste se reprodujo, sin determinar obstrucción gástrica. Por reaparición de sintomatología se realizó una gastrectomía subtotal en Y de Roux por laparoscopía (Figura 7) con cuatro días de hospitalización y evolución sin complicaciones. El estudio histopatológico demostró una lesión quística, cuya pared no presentaba revestimiento y este formada por tejido fibroso, colágeno e infiltrado inflamatorio de predominio linfohistiocitario. En la periferia del quiste se observó tejido pancreático heterotópico, en el espesor de la pared gástrica, formado por porciones variables de conductos y acinos de estructura conservada (Figura 8).

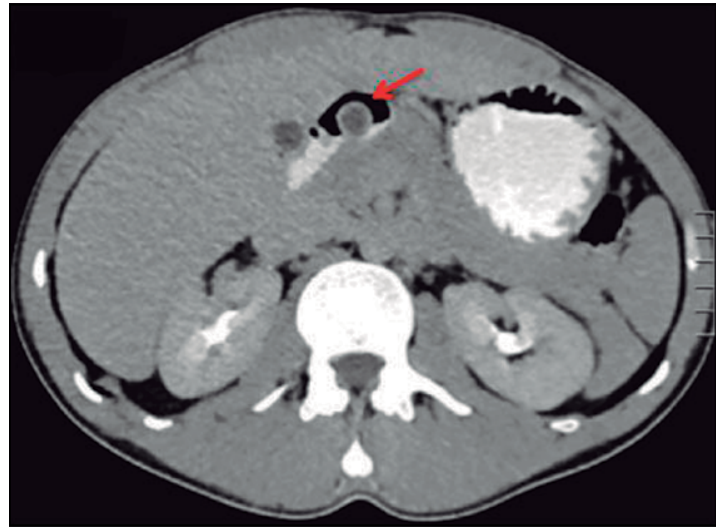

Figura 6. Imagen axial de tomografía computada con contraste oral $24 \mathrm{~h}$ post-drenaje demostrando adecuado paso del medio de contraste al intestino delgado e imagen residual de $18 \mathrm{~mm}$ (flecha roja).

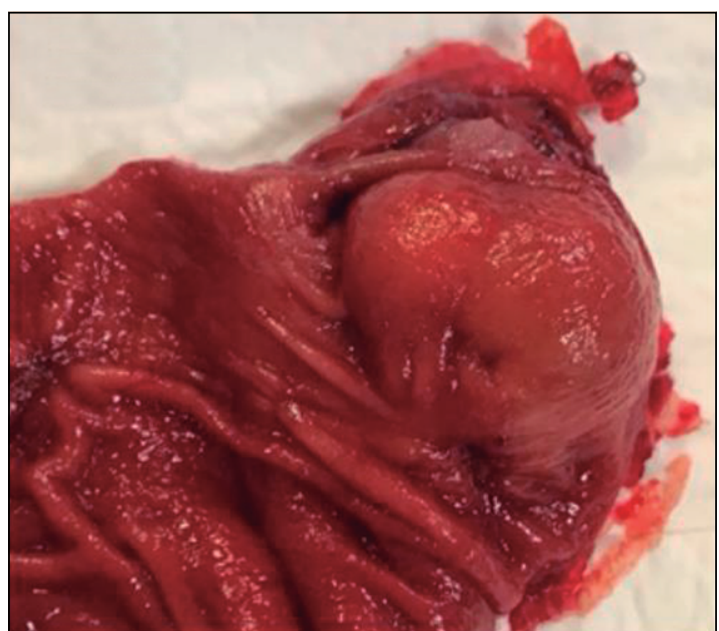

Figura 7. Pieza quirúrgica. Pseudoquiste en pared del antro gástrico. 


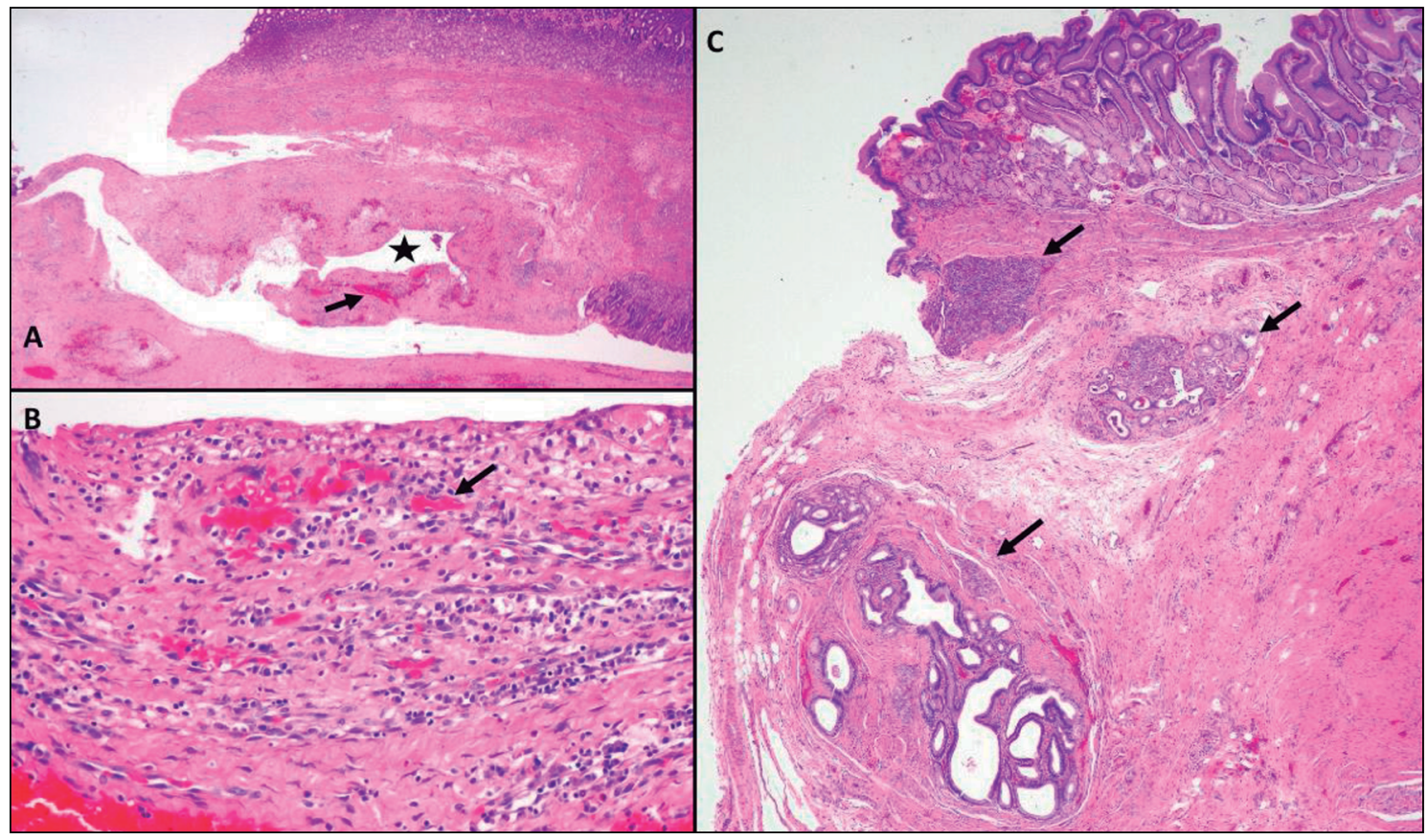

Figura 8. A: Tinción HE, 2X: Pared gástrica con lesión quística(estrella) sin revestimiento epitelial, con focos de hemorragia (flechas). B: 20X: mayor detalle de la pared del quiste, formada por linfocitos, plasmocitos e histiocitos, muchos con citoplasma espumoso y focos de hemorragia (flecha). C: Tinción HE, 5X: Pared gástrica revestida por mucosa antropilórica, con tres acinos pancreáticos en su espesor (flechas), predominantemente en la submucosa, dos de ellos con conductos dilatados.

\section{Discusión}

Los pseudoquistes intramurales del tracto intestinal son de muy rara incidencia y el mecanismo exacto de su formación no es bien conocido. Los posibles mecanismos para su formación son rotura de un pseudoquiste pancreático dentro de la pared gastrointestinal, presencia de una fístula entre el páncreas y el tubo digestivo o inflamación de tejido pancreático heterotópico dentro de la pared gastrointestinal ${ }^{4,5}$.

El páncreas heterotópico es una anomalía congénita en la cual el tejido pancreático está anatómicamente separado de la glándula princi$\mathrm{pal}^{6}$. Las localizaciones más comunes incluyen el tracto gastrointestinal superior, especialmente el estómago, duodeno y yeyuno proximal. El tejido pancreático heterotópico gástrico típicamente tiene una localización antral y un patrón de crecimiento endoluminal, sin deformar el contorno externo de la pared ${ }^{7}$.

La apariencia tomográfica más común del páncreas heterotópico es una pequeña masa in- tramural ovalada, con márgenes microlobulados y crecimiento endoluminal que puede ser confundida con un engrosamiento parietal neoplásico ${ }^{1}$.

El tejido pancreático heterotópico no complicado es generalmente asintomático. Las complicaciones de este incluyen pancreatitis, formación de pseudoquistes, degeneración maligna, sangrado gastrointestinal, obstrucción intestinal e invaginación intestinal ${ }^{8}$.

Debido a la rara frecuencia de pseudoquistes pancreáticos intramurales gástricos, son de difícil diagnóstico y además representan un dilema terapéutico para el clínico. El diagnóstico de un pseudoquiste pancreático intramural gástrico puede ser realizado por tomografía computada y endosonografía ${ }^{9}$. La aspiración de líquido con alto contenido en amilasa y lipasa es una manera confiable para confirmar el diagnóstico ${ }^{10}$. Su manejo se ha basado en reportes de casos, la mayoría de los cuales han sido tratados tanto mediante cirugía como con drenaje percutáneo o endoscópico y, en algunos casos asintomáticos el manejo ha sido terapia médica conservadora y conducta 
expectante $2,11,12$. En Chile se han reportado casos aislados de tejido pancreático ectópico como hallazgo incidental en estudios histológicos gástricos de pacientes obesos mórbidos sometidos a gastrectomía vertical y de lesiones submucosas gastroesofágicas sometidas a biopsia, en ambos reportes se encontró solo un caso, ninguno de los cuales se presentó asociado a síntomas como el nuestro ${ }^{13,14}$.

\section{Conclusión}

Se presenta un caso de síndrome de retención gástrica secundario a un pseudoquiste pancreático intramural gástrico en un páncreas heterotópico. Corresponde a una patología infrecuente, con pocos casos reportados. En nuestro caso, el diagnóstico y manejo endosonográfico logró aliviar los síntomas con una evolución libre de complicaciones y sin recurrencia de los síntomas. Los pseudoquistes intramurales gástricos que se presentan con dolor abdominal y síntomas de obstrucción gástrica son muy raros y la endosonografía es una modalidad diagnóstica y terapéutica de gran utilidad. El reconocimiento de las características imagenológicas del tejido pancreático heterotópico y sus complicaciones ayuda a diferenciar este de otras lesiones, especialmente neoplásicas y evitar cirugías innecesarias.

\section{Referencias}

1. Rezvani M, Menias C, Sandrasegaran K, Olpin JD, Elsayes KM, Shaaban AM. Heterotopic Pancreas: Histopathologic Features, Imaging Findings, and Complications. RadioGraphics 2017; 37 (2): 484-99.

2. Bhasin DK, Rana SS, Nanda M, Chandail VS, Masoodi I, Kang M, et al. Endoscopic management of pancreatic pseudocysts at atypical locations. Surg Endosc 2010; 24 (5): 1085-91.

3. Claudon M, Verain AL, Bigard MA, Boissel P, Poisson
$\mathrm{P}$, Floquet J, et al. Cyst formation in gastric heterotopic pancreas: report of two cases. Radiology 1988; 169: 65960.

4. Rana SS, Bhasin DK, Rao C, Gupta R. Intramural pseudocysts of the upper gastrointestinal tract. Endosc Ultrasound 2013; 2 (4): 194-8.

5. Eisenberger CF, Kropp A, Langwieler TE, Gocht A, Izbicki JR, Knoefel WT. Heterotopic pancreatitis: gastric outlet obstruction due to an intramural pseudocyst and hamartoma. Z Gastroenterol 2002; 40 (4): 259-62.

6. Trifan A, Târcoveanu E, Danciu M, Huţanaşu C, Cojocariu C, Stanciu C. Gastric heterotopic pancreas: An unusual case and review of the literature. J Gastrointest Liver Dis 2012; 21 (2): 209-12.

7. Christodoulidis G, Zacharoulis D, Barbanis S, Katsogridakis E, Hatzitheofilou K. Heterotopic pancreas in the stomach: A case report and literature review. World J Gastroenterol 2007; 13 (45): 6098-100.

8. Shaib YH, Rabaa E, Feddersen RM, Jamal MM, Qaseem T. Gastric outlet obstruction secondary to heterotopic pancreas in the antrum: Case report and review. Gastrointest Endosc 2001; 54 (4): 527-30.

9. Muller U, Schachschal G, Voderholzer WA. Image of the month. Gastroenterology 2005; 129 (1): 1-2.

10. Chiu HH, Liu YW, Li JH. Gastric Intramural Pseudocyst Presenting as a Gastric Submucosal Lesion. Clin Gastroenterol Hepatol 2007; 5 (6): 20-1.

11. Luigiano C, Cennamo V, Ferrara F, Fabbri C. Endoscopic ultrasound-fine needle aspiration diagnosis and treatment of a gastric intramural pseudocyst. Dig Liver Dis 2012; 44 (8): 8658.

12. Matsumoto T, Tanaka N, Nagai M, Koike D, Sakuraoka Y, Kubota K. A case of gastric heterotopic pancreatitis resected by laparoscopic surgery. Int Surg 2015; 100 (4): 678-82.

13. Burgos LAM, Csendes JA, Braghetto MI, Muñoz CHA, Villanueva IM. Hallazgos histológicos gástricos en obesos morbidos sometidos a gastrectomía vertical laparoscópica. Rev Chil Cir 2014; 66 (3): 224-30.

14. Cortés GP, Pavez OC, Méndez AL, Alvarez LM, Ibáñez LP. Técnica de incisión submucosa para estudio histológico de lesiones subepiteliales gastroesofágicas. Gastroenterol. latinoam 2010; 21 (4): 468-75. 